\title{
Admissible classes of multivalent functions associated with an integral operator
}

\begin{abstract}
In this paper we investigate some applications of the differential subordination and superordination of classes of admissible functions associated with an integral operator. Additionally, differential sandwich-type results are obtained.
\end{abstract}

1. Introduction. Let $\mathcal{H}(\mathbb{U})$ be the class of functions analytic in the disk $\mathbb{U}=\{z \in \mathbb{C}:|z|<1\}$ and $\mathcal{H}[a, n]$ be the subclass of $\mathcal{H}(\mathbb{U})$ consisting of functions of the form:

$$
f(z)=a+a_{n} z^{n}+a_{n+1} z^{n+1}+\ldots .
$$

Let $f$ and $F$ be members of $\mathcal{H}(\mathbb{U})$, the function $f(z)$ is said to be subordinate to $F(z)$, or $F(z)$ is said to be superordinate to $f(z)$, if there exists a function $\omega(z)$ analytic in $\mathbb{U}$ with $\omega(0)=0$ and $|\omega(z)|<1, z \in \mathbb{U}$, such that $f(z)=$ $F(\omega(z))$. In such a case we write $f(z) \prec F(z)$. If $F$ is univalent, then $f(z) \prec F(z)$ if and only if $f(0)=F(0)$ and $f(\mathbb{U}) \subset F(\mathbb{U})$ (see $[7,10]$ and $[11])$.

Let $\phi: \mathbb{C}^{3} \times \mathbb{U} \rightarrow \mathbb{C}$ and $h(z)$ be univalent in $\mathbb{U}$. If $p(z)$ is analytic in $\mathbb{U}$ and satisfies the second order differential subordination:

$$
\phi\left(p(z), z p^{\prime}(z), z^{2} p^{\prime \prime}(z) ; z\right) \prec h(z),
$$

2010 Mathematics Subject Classification. Primary 30C45. Secondary 30D30, 33D20.

Key words and phrases. Analytic function, superordination, sandwich-type, admissible class, integral operator. 
then $p(z)$ is a solution of the differential subordination (1.1). The univalent function $q(z)$ is called a dominant of the solutions of (1.1) if $p(z) \prec q(z)$ for all $p(z)$ satisfying (1.1). A univalent dominant $\tilde{q}$ that satisfies $\tilde{q} \prec q$ for all dominants of (1.1) is called the best dominant.

If $p(z)$ and $\phi\left(p(z), z p^{\prime}(z), z^{2} p^{\prime \prime}(z) ; z\right)$ are univalent in $\mathbb{U}$ and if $p(z)$ satisfies second order differential superordination:

$$
h(z) \prec \phi\left(p(z), z p^{\prime}(z), z^{2} p^{\prime \prime}(z) ; z\right),
$$

then $p(z)$ is a solution of the differential superordination (1.2). An analytic function $q(z)$ is called a subordinant of the solutions of (1.2) if $q(z) \prec p(z)$ for all $p(z)$ satisfying (1.2). A univalent subordinant $\tilde{q}$ that satisfies $q \prec \tilde{q}$ for all subordinants of (1.2) is called the best subordinant.

Let $\mathcal{A}(p)$ denote the class of all analytic functions and $p$-valent of the form:

$$
f(z)=z^{p}+\sum_{n=p+1}^{\infty} a_{n} z^{n} \quad(p \in \mathbb{N}=\{1,2,3, \ldots\} ; z \in \mathbb{U}) .
$$

Motivated essentially by Jung et al. [9], Shams et al. [12] introduced the integral operator $I_{p}^{\alpha}: \mathcal{A}(p) \rightarrow \mathcal{A}(p)$ as follows:

$$
I_{p}^{\alpha} f(z)= \begin{cases}\frac{(p+1)^{\alpha}}{z \Gamma(\alpha)} \int_{0}^{z}\left(\log \frac{z}{t}\right)^{\alpha-1} f(t) d t & (\alpha>0) \\ f(z) & (\alpha=0) .\end{cases}
$$

For $f \in \mathcal{A}(p)$ given by (1.3), then from (1.4), we deduce that

$$
I_{p}^{\alpha} f(z)=z^{p}+\sum_{n=p+1}^{\infty}\left(\frac{p+1}{n+1}\right)^{\alpha} a_{n} z^{n} \quad(\alpha \geq 0 ; p \in \mathbb{N}) .
$$

It is easily verified from (1.5) that

$$
z\left(I_{p}^{\alpha} f(z)\right)^{\prime}=(p+1) I_{p}^{\alpha-1} f(z)-I_{p}^{\alpha} f(z) .
$$

We note that the integral operator $I_{1}^{\alpha}=I^{\alpha}$ was defined by Jung et al. [9].

To prove our results, we need the following definitions and lemmas.

Denote by $\mathcal{F}$ the set of all functions $q$ that are analytic and injective on $\overline{\mathbb{U}} \backslash E(q)$, where

$$
E(q)=\left\{\zeta \in \partial \mathbb{U}: \lim _{z \rightarrow \zeta} q(z)=\infty\right\}
$$

and are such that $q^{\prime}(\zeta) \neq 0$ for $\zeta \in \partial \mathbb{U} \backslash E(q)$. Further let the subclass of $\mathcal{F}$ for which $q(0)=a$ be denoted by $\mathcal{F}(a)$ and $\mathcal{F}(0) \equiv \mathcal{F}_{0}$.

In order to prove our results, we shall make use of the following classes of admissible functions. 
Definition 1.1 ([10, Definition 2.3a, p. 27]). Let $\Omega$ be a set in $\mathbb{C}, q \in \mathcal{F}$ and $n$ be a positive integer. The class of admissible functions $\Psi_{n}[\Omega, q]$, consists of those functions $\psi: \mathbb{C}^{3} \times \mathbb{U} \rightarrow \mathbb{C}$ that satisfy the admissibility condition

$$
\psi(r, s, t ; z) \notin \Omega
$$

whenever

$$
r=q(\zeta), s=k \zeta q^{\prime}(\zeta), \Re\left\{\frac{t}{s}+1\right\} \geq k \Re\left\{1+\frac{\zeta q^{\prime \prime}(\zeta)}{q^{\prime}(\zeta)}\right\},
$$

where $z \in \mathbb{U}, \zeta \in \partial \mathbb{U} \backslash E(q)$ and $k \geq n$. We write $\Psi_{1}[\Omega, q]$ as $\Psi[\Omega, q]$.

In particular, if

$$
q(z)=M \frac{M z+a}{M+\bar{a} z} \quad(M>0,|a|<M),
$$

then $q(\mathbb{U})=\mathbb{U}_{M}=\{w:|w|<M\}, q(0)=a, E(q)=\emptyset$ and $q \in \mathcal{F}(a)$. In this case, we set $\Psi_{n}[\Omega, M, a]=\Psi_{n}[\Omega, q]$, and in the special case when the set $\Omega=\mathbb{U}_{M}$, the class is simply denoted by $\Psi_{n}[M, a]$.

Definition 1.2 ([11, Definition 3, p. 817]). Let $\Omega$ be a set in $\mathbb{C}, q \in \mathcal{H}[a, n]$ with $q^{\prime}(z) \neq 0$. The class of admissible functions $\Psi_{n}^{\prime}[\Omega, q]$ consists of those functions $\psi: \mathbb{C}^{3} \times \overline{\mathbb{U}} \rightarrow \mathbb{C}$ that satisfy the admissibility condition

$$
\psi(r, s, t ; \zeta) \in \Omega
$$

whenever

$$
r=q(z), \quad s=\frac{z q^{\prime}(z)}{m}, \Re\left\{\frac{t}{s}+1\right\} \leq \frac{1}{m} \Re\left\{1+\frac{z q^{\prime \prime}(z)}{q^{\prime}(z)}\right\},
$$

where $z \in \mathbb{U}, \zeta \in \partial \mathbb{U}$ and $m \geq n \geq 1$. In particular, we write $\Psi_{1}^{\prime}[\Omega, q]$ as $\Psi^{\prime}[\Omega, q]$.

In our investigation we need the following lemmas which are proved by Miller and Mocanu [10] and [11].

Lemma 1.3 ([10, Theorem 2.3b, p. 28]). Let $\psi \in \Psi_{n}[\Omega, q]$ with $q(0)=a$. If the analytic function $g(z)=a+a_{n} z^{n}+a_{n+1} z^{n+1}+\ldots$ satisfies

$$
\psi\left(g(z), z g^{\prime}(z), z^{2} g^{\prime \prime}(z) ; z\right) \in \Omega
$$

then $g \prec q$.

Lemma 1.4 ([11, Theorem 1, p. 818]). Let $\psi \in \Psi_{n}^{\prime}[\Omega, q]$ with $q(0)=a$. If $g \in \mathcal{F}(a)$ and

$$
\psi\left(g(z), z g^{\prime}(z), z^{2} g^{\prime \prime}(z) ; z\right)
$$

is univalent in $\mathbb{U}$, then

implies $q \prec g$.

$$
\Omega \subset\left\{\psi\left(g(z), z g^{\prime}(z), z^{2} g^{\prime \prime}(z) ; z\right): z \in \mathbb{U}\right\},
$$


In particular, Aouf and Seoudy [6] investigated a subordination and superordination problems for multivalent functions defined by the integral operator $I_{p}^{\alpha}$, they have determined classes of admissible functions so that

$$
q_{1}(z) \prec I_{p}^{\alpha} f(z) \prec q_{2}(z)
$$

and

$$
q_{1}(z) \prec \frac{I_{p}^{\alpha} f(z)}{z^{p-1}} \prec q_{2}(z),
$$

where $q_{1}$ and $q_{2}$ are given univalent functions in $\mathbb{U}$.

In this paper, we determine the sufficient conditions for certain classes of admissible functions of multivalent functions associated with $I_{p}^{\alpha}$ so that

$$
q_{1}(z) \prec\left[I_{p}^{\alpha} f(z)\right]^{\mu} \prec q_{2}(z)
$$

and

$$
q_{1}(z) \prec\left[\frac{I_{p}^{\alpha} f(z)}{z^{p-1}}\right]^{\mu} \prec q_{2}(z),
$$

where $\mu>0$ and $q_{1}$ and $q_{2}$ are given univalent functions in $\mathbb{U}$. Additionally, differential sandwich-type results are obtained. A similar problem for analytic functions was studied by Aghalary et al. [1], Ali et al. [2], Aouf et al. [4], and Kim and Srivastava [8] and others (see [3, 5] and [6]).

2. Subordination results involving $\boldsymbol{I}_{p}^{\alpha}$. Unless otherwise mentioned, we assume throughout this paper that $\alpha>2, \mu>0, p \in \mathbb{N}, z \in \mathbb{U}$ and all powers are principal ones.

Definition 2.1. Let $\Omega$ be a set in $\mathbb{C}$ and $q \in \mathcal{F}_{0} \cap \mathcal{H}[0, \mu p]$. The class of admissible functions $\Phi_{1}[\Omega, q, \mu]$ consists of those functions $\phi: \mathbb{C}^{3} \times \mathbb{U} \rightarrow \mathbb{C}$ that satisfy the admissibility condition

$$
\phi(u, v, w ; z) \notin \Omega
$$

whenever

$$
\begin{gathered}
u=q(\zeta), v=\frac{k \zeta q^{\prime}(\zeta)+\mu q(\zeta)}{\mu(p+1)}, \\
\Re\left\{\frac{(p+1)^{2} w-2 \mu(p+1) v+\mu u}{(p+1) v-u}\right\} \geq k \Re\left\{1+\frac{\zeta q^{\prime \prime}(\zeta)}{q^{\prime}(\zeta)}\right\},
\end{gathered}
$$

where $z \in \mathbb{U}, \zeta \in \partial \mathbb{U} \backslash E(q)$ and $k \geq \mu p$.

Theorem 2.2. Let $\phi \in \Phi_{1}[\Omega, q, \mu]$. If $f \in \mathcal{A}(p)$ satisfies

$$
\begin{gathered}
\left\{\phi \left(\left[I_{p}^{\alpha} f(z)\right]^{\mu},\left[I_{p}^{\alpha} f(z)\right]^{\mu-1} I_{p}^{\alpha-1} f(z),\left[I_{p}^{\alpha} f(z)\right]^{\mu-1} I_{p}^{\alpha-2} f(z)\right.\right. \\
\left.\left.+(\mu-1)\left[I_{p}^{\alpha} f(z)\right]^{\mu-2}\left[I_{p}^{\alpha-1} f(z)\right]^{2} ; z\right): z \in \mathbb{U}\right\} \subset \Omega
\end{gathered}
$$

then

$$
\left[I_{p}^{\alpha} f(z)\right]^{\mu} \prec q(z) .
$$


Proof. Define the analytic function $g(z)$ in $\mathbb{U}$ by

$$
g(z)=\left[I_{p}^{\alpha} f(z)\right]^{\mu} .
$$

In view of the relation (1.6), from (2.2) we get

$$
\left[I_{p}^{\alpha} f(z)\right]^{\mu-1} I_{p}^{\alpha-1} f(z)=\frac{z g^{\prime}(z)+\mu g(z)}{\mu(p+1)} .
$$

Further computations show that

$$
\begin{gathered}
{\left[I_{p}^{\alpha} f(z)\right]^{\mu-1} I_{p}^{\alpha-2} f(z)+(\mu-1)\left[I_{p}^{\alpha} f(z)\right]^{\mu-2}\left[I_{p}^{\alpha-1} f(z)\right]^{2}} \\
=\frac{z^{2} g^{\prime \prime}(z)+(2 \mu+1) z g^{\prime}(z)+\mu^{2} g(z)}{\mu(p+1)^{2}} .
\end{gathered}
$$

Define the transformations from $\mathbb{C}^{3}$ to $\mathbb{C}$ by

$$
u=r, v=\frac{s+\mu r}{\mu(p+1)}, \quad w=\frac{t+(2 \mu+1) s+\mu^{2} r}{\mu(p+1)^{2}} .
$$

Let

$$
\begin{aligned}
\psi(r, s, t ; z) & =\phi(u, v, w ; z) \\
& =\phi\left(r, \frac{s+\mu r}{\mu(p+1)}, \frac{t+(2 \mu+1) s+\mu^{2} r}{\mu(p+1)^{2}} ; z\right) .
\end{aligned}
$$

Using (2.2)-(2.6), we obtain

$$
\begin{gathered}
\psi\left(g(z), z g^{\prime}(z), z^{2} g^{\prime \prime}(z) ; z\right)=\phi\left(\left[I_{p}^{\alpha} f(z)\right]^{\mu},\left[I_{p}^{\alpha} f(z)\right]^{\mu-1} I_{p}^{\alpha-1} f(z),\right. \\
\left.\left[I_{p}^{\alpha} f(z)\right]^{\mu-1} I_{p}^{\alpha-2} f(z)+(\mu-1)\left[I_{p}^{\alpha} f(z)\right]^{\mu-2}\left[I_{p}^{\alpha-1} f(z)\right]^{2} ; z\right) .
\end{gathered}
$$

Hence (2.1) becomes

$$
\psi\left(g(z), z g^{\prime}(z), z^{2} g^{\prime \prime}(z) ; z\right) \in \Omega .
$$

The proof is completed if it can be shown that the admissibility condition for $\phi \in \Phi_{1}[\Omega, q, \mu]$ is equivalent to the admissibility condition for $\psi$ given in Definition 1.1. Note that

$$
\frac{t}{s}+1=\frac{(p+1)^{2} w-2 \mu(p+1) v+\mu u}{(p+1) v-u},
$$

and hence $\psi \in \Psi_{\mu p}[\Omega, q]$. By Lemma 1.3,

$$
g(z) \prec q(z) \quad \text { or } \quad\left[I_{p}^{\alpha} f(z)\right]^{\mu} \prec q(z) .
$$

If $\Omega \neq \mathbb{C}$ is a simply connected domain, then $\Omega=h(\mathbb{U})$ for some conformal mapping $h$ of $\mathbb{U}$ onto $\Omega$. In this case the class $\Phi_{1}[h(\mathbb{U}), q, \mu]$ is written as $\Phi_{1}[h, q, \mu]$. The following result is an immediate consequence of Theorem 2.2. 
Theorem 2.3. Let $\phi \in \Phi_{1}[h, q, \mu]$. If $f \in \mathcal{A}(p)$ satisfies

$$
\begin{gathered}
\phi\left(\left[I_{p}^{\alpha} f(z)\right]^{\mu},\left[I_{p}^{\alpha} f(z)\right]^{\mu-1} I_{p}^{\alpha-1} f(z),\left[I_{p}^{\alpha} f(z)\right]^{\mu-1} I_{p}^{\alpha-2} f(z)\right. \\
\left.+(\mu-1)\left[I_{p}^{\alpha} f(z)\right]^{\mu-2}\left[I_{p}^{\alpha-1} f(z)\right]^{2} ; z\right) \prec h(z),
\end{gathered}
$$

then

$$
\left[I_{p}^{\alpha} f(z)\right]^{\mu} \prec q(z) .
$$

Our next result is an extension of Theorem 2.2 to the case where the behavior of $q$ on $\partial \mathbb{U}$ is not known.

Corollary 2.4. Let $\Omega \subset \mathbb{C}$ and let $q$ be univalent in $\mathbb{U}, q(0)=0$. Let $\phi \in \Phi_{1}\left[\Omega, q_{\rho}, \mu\right]$ for some $\rho \in(0,1)$, where $q_{\rho}(z)=q(\rho z)$. If $f \in \mathcal{A}(p)$ and

$$
\begin{gathered}
\phi\left(\left[I_{p}^{\alpha} f(z)\right]^{\mu},\left[I_{p}^{\alpha} f(z)\right]^{\mu-1} I_{p}^{\alpha-1} f(z),\left[I_{p}^{\alpha} f(z)\right]^{\mu-1} I_{p}^{\alpha-2} f(z)\right. \\
\left.+(\mu-1)\left[I_{p}^{\alpha} f(z)\right]^{\mu-2}\left[I_{p}^{\alpha-1} f(z)\right]^{2} ; z\right) \in \Omega
\end{gathered}
$$

then

$$
\left[I_{p}^{\alpha} f(z)\right]^{\mu} \prec q(z) .
$$

Proof. Theorem 2.2 yields $\left[I_{p}^{\alpha} f(z)\right]^{\mu} \prec q_{\rho}(z)$. The result is now deduced from $q_{\rho}(z) \prec q(z)$.

Theorem 2.5. Let $h$ and $q$ be univalent in $\mathbb{U}$, with $q(0)=0$ and set $q_{\rho}(z)=$ $q(\rho z)$ and $h_{\rho}(z)=h(\rho z)$. Let $\phi: \mathbb{C}^{3} \times \mathbb{U} \rightarrow \mathbb{C}$ satisfy one of the following conditions:

(1) $\phi \in \Phi_{1}\left[h, q_{\rho}, \mu\right]$, for some $\rho \in(0,1)$, or

(2) there exists $\rho_{0} \in(0,1)$ such that $\phi \in \Phi_{1}\left[h_{\rho}, q_{\rho}, \mu\right]$, for all $\rho \in\left(\rho_{0}, 1\right)$.

If $f \in \mathcal{A}(p)$ satisfies (2.8), then

$$
\left[I_{p}^{\alpha} f(z)\right]^{\mu} \prec q(z) .
$$

Proof. The proof is similar to the proof of [10, Theorem 2.3d, p. 30] and is therefore omitted.

The next theorem yields the best dominant of the differential subordination (2.8).

Theorem 2.6. Let $h$ be univalent in $\mathbb{U}$. Let $\phi: \mathbb{C}^{3} \times \mathbb{U} \rightarrow \mathbb{C}$. Suppose that the differential equation

$$
\phi\left(q(z), z q^{\prime}(z), z^{2} q^{\prime \prime}(z) ; z\right)=h(z)
$$

has a solution $q$ such that $q(0)=0$ and satisfies one of the following conditions:

(1) $q \in \mathcal{F}_{0}$ and $\phi \in \Phi_{1}[h, q, \mu]$,

(2) $q$ is univalent in $\mathbb{U}$ and $\phi \in \Phi_{1}\left[h, q_{\rho}, \mu\right]$, for some $\rho \in(0,1)$, or 
(3) $q$ is univalent in $\mathbb{U}$ and there exists $\rho_{0} \in(0,1)$ such that $\phi \in$ $\Phi_{1}\left[h_{\rho}, q_{\rho}, \mu\right]$, for all $\rho \in\left(\rho_{0}, 1\right)$.

If $f \in \mathcal{A}(p)$ satisfies (2.8), then

$$
\left[I_{p}^{\alpha} f(z)\right]^{\mu} \prec q(z),
$$

and $q$ is the best dominant.

Proof. Following the same arguments in [10, Theorem 2.3e, p. 31], we deduce that $q$ is a dominant from Theorems 2.3 and 2.5. Since $q$ satisfies (2.9) it is also a solution of (2.8) and therefore $q$ will be dominated by all dominants. Hence $q$ is the best dominant.

In the particular case $q(z)=M z, M>0$, and in view of the Definition 2.1, the class of admissible functions $\Phi_{1}[\Omega, q, \mu]$, denoted by $\Phi_{1}[\Omega, M, \mu]$, is described below.

Definition 2.7. Let $\Omega$ be a set in $\mathbb{C}$ and $M>0$. The class of admissible functions $\Phi_{1}[\Omega, M, \mu]$ consists of those functions $\phi: \mathbb{C}^{3} \times \mathbb{U} \rightarrow \mathbb{C}$ such that

$$
\phi\left(M e^{i \theta}, \frac{k+\mu}{\mu(p+1)} M e^{i \theta}, \frac{L+\left[(2 \mu+1) k+\mu^{2}\right] M e^{i \theta}}{\mu(p+1)^{2}} ; z\right) \notin \Omega
$$

whenever $z \in \mathbb{U}, \theta \in \mathbb{R}, \Re\left(L e^{-i \theta}\right) \geq(k-1) k M$ for all real $\theta$ and $k \geq \mu p$.

Corollary 2.8. Let $\phi \in \Phi_{1}[\Omega, M, \mu]$. If $f \in \mathcal{A}(p)$ satisfies

$$
\begin{gathered}
\phi\left(\left[I_{p}^{\alpha} f(z)\right]^{\mu},\left[I_{p}^{\alpha} f(z)\right]^{\mu-1} I_{p}^{\alpha-1} f(z),\left[I_{p}^{\alpha} f(z)\right]^{\mu-1} I_{p}^{\alpha-2} f(z)\right. \\
\left.+(\mu-1)\left[I_{p}^{\alpha} f(z)\right]^{\mu-2}\left[I_{p}^{\alpha-1} f(z)\right]^{2} ; z\right) \in \Omega
\end{gathered}
$$

then

$$
\left|I_{p}^{\alpha} f(z)\right|^{\mu}<M \quad(z \in \mathbb{U}) .
$$

In the special case $\Omega=q(\mathbb{U})=\{\omega:|\omega|<M\}$, the class $\Phi_{1}[\Omega, M, \mu]$ is simply denoted by $\Phi_{1}[M, \mu]$.

Corollary 2.9. Let $\phi \in \Phi_{1}[M, \mu]$. If $f \in \mathcal{A}(p)$ satisfies

$$
\begin{aligned}
\mid \phi( & {\left[I_{p}^{\alpha} f(z)\right]^{\mu},\left[I_{p}^{\alpha} f(z)\right]^{\mu-1} I_{p}^{\alpha-1} f(z),\left[I_{p}^{\alpha} f(z)\right]^{\mu-1} I_{p}^{\alpha-2} f(z) } \\
& \left.+(\mu-1)\left[I_{p}^{\alpha} f(z)\right]^{\mu-2}\left[I_{p}^{\alpha-1} f(z)\right]^{2} ; z\right) \mid<M
\end{aligned}
$$

then

$$
\left|I_{p}^{\alpha} f(z)\right|^{\mu}<M
$$

Remark 2.10. Putting $\mu=M=1$ in the Corollary 2.9, we obtain the result obtained by Aouf [3, Theorem 1]. 
Corollary 2.11. If $k \geq \mu p$ and $f \in \mathcal{A}(p)$ satisfies

$$
\left|\left[I_{p}^{\alpha} f(z)\right]^{\mu-1} I_{p}^{\alpha-1} f(z)\right|<M,
$$

then

$$
\left|I_{p}^{\alpha} f(z)\right|^{\mu}<M
$$

Proof. This follows from Corollary 2.9 by taking

$$
\phi(u, v, w ; z)=v=\frac{k+\mu}{\mu(p+1)} M e^{i \theta} .
$$

Remark 2.12. For $\mu=M=1$, Corollary 2.11 yields the result obtained by Aouf [3, Corollary 1].

Definition 2.13. Let $\Omega$ be a set in $\mathbb{C}$ and $q \in \mathcal{F}_{0} \cap \mathcal{H}[0, \mu]$. The class of admissible functions $\Phi_{2}[\Omega, q, \mu]$ consists of those functions $\phi: \mathbb{C}^{3} \times \mathbb{U} \rightarrow \mathbb{C}$ that satisfy the admissibility condition

$$
\phi(u, v, w ; z) \notin \Omega
$$

whenever

$$
\begin{gathered}
u=q(\zeta), v=\frac{k \zeta q^{\prime}(\zeta)+\mu p q(\zeta)}{\mu(p+1)}, \\
\Re\left\{\frac{(p+1)^{2} w-2 \mu p(p+1) v+\mu p^{2} u}{(p+1) v-p u}\right\} \geq k \Re\left\{1+\frac{\zeta q^{\prime \prime}(\zeta)}{q^{\prime}(\zeta)}\right\},
\end{gathered}
$$

where $z \in \mathbb{U}, \zeta \in \partial \mathbb{U} \backslash E(q)$ and $k \geq \mu$.

Theorem 2.14. Let $\phi \in \Phi_{2}[\Omega, q, \mu]$. If $f \in \mathcal{A}(p)$ satisfies

$$
\begin{gathered}
\left\{\phi \left(\left[\frac{I_{p}^{\alpha} f(z)}{z^{p-1}}\right]^{\mu},\left[\frac{I_{p}^{\alpha} f(z)}{z^{p-1}}\right]^{\mu} \frac{I_{p}^{\alpha-1} f(z)}{I_{p}^{\alpha} f(z)},\left[\frac{I_{p}^{\alpha} f(z)}{z^{p-1}}\right]^{\mu} \frac{I_{p}^{\alpha-2} f(z)}{I_{p}^{\alpha} f(z)}\right.\right. \\
\left.\left.\quad+(\mu-1)\left[\frac{I_{p}^{\alpha} f(z)}{z^{p-1}}\right]^{\mu}\left[\frac{I_{p}^{\alpha-1} f(z)}{I_{p}^{\alpha} f(z)}\right]^{2} ; z\right): z \in \mathbb{U}\right\} \subset \Omega,
\end{gathered}
$$

then

$$
\left[\frac{I_{p}^{\alpha} f(z)}{z^{p-1}}\right]^{\mu} \prec q(z) .
$$

Proof. Define an analytic function $g$ in $\mathbb{U}$ by

$$
g(z)=\left[\frac{I_{p}^{\alpha} f(z)}{z^{p-1}}\right]^{\mu} \quad(z \in \mathbb{U}) .
$$

By making use of (1.6) and (2.12), we get

$$
\left[\frac{I_{p}^{\alpha} f(z)}{z^{p-1}}\right]^{\mu} \frac{I_{p}^{\alpha-1} f(z)}{I_{p}^{\alpha} f(z)}=\frac{z g^{\prime}(z)+\mu p g(z)}{\mu(p+1)} .
$$


Further computations show that

$$
\begin{gathered}
{\left[\frac{I_{p}^{\alpha} f(z)}{z^{p-1}}\right]^{\mu} \frac{I_{p}^{\alpha-2} f(z)}{I_{p}^{\alpha} f(z)}+(\mu-1)\left[\frac{I_{p}^{\alpha} f(z)}{z^{p-1}}\right]^{\mu}\left[\frac{I_{p}^{\alpha-1} f(z)}{I_{p}^{\alpha} f(z)}\right]^{2}} \\
=\frac{z^{2} g^{\prime \prime}(z)+(1+2 \mu p) z g^{\prime}(z)+\mu^{2} p^{2} g(z)}{\mu(p+1)^{2}}
\end{gathered}
$$

Define the transformations from $\mathbb{C}^{3}$ to $\mathbb{C}$ by

$$
\begin{aligned}
& u=r, v=\frac{s+\mu p r}{\mu(p+1)}, \\
& w=\frac{t+(1+2 \mu p) s+\mu^{2} p^{2} r}{\mu(p+1)^{2}} .
\end{aligned}
$$

Let

$$
\begin{aligned}
\psi(r, s, t ; z) & =\phi(u, v, w ; z) \\
& =\phi\left(r, \frac{s+\mu p r}{\mu(p+1)}, \frac{t+(1+2 \mu p) s+\mu^{2} p^{2} r}{\mu(p+1)^{2}} ; z\right) .
\end{aligned}
$$

Using (2.12)-(2.16), we obtain

$$
\begin{aligned}
\psi( & \left.g(z), z g^{\prime}(z), z^{2} g^{\prime \prime}(z) ; z\right) \\
= & \phi\left(\left[\frac{I_{p}^{\alpha} f(z)}{z^{p-1}}\right]^{\mu},\left[\frac{I_{p}^{\alpha} f(z)}{z^{p-1}}\right]^{\mu} \frac{I_{p}^{\alpha-1} f(z)}{I_{p}^{\alpha} f(z)},\right. \\
& {\left.\left[\frac{I_{p}^{\alpha} f(z)}{z^{p-1}}\right]^{\mu} \frac{I_{p}^{\alpha-2} f(z)}{I_{p}^{\alpha} f(z)}+(\mu-1)\left[\frac{I_{p}^{\alpha} f(z)}{z^{p-1}}\right]^{\mu}\left[\frac{I_{p}^{\alpha-1} f(z)}{I_{p}^{\alpha} f(z)}\right]^{2} ; z\right) . }
\end{aligned}
$$

Hence (2.11) becomes

$$
\psi\left(g(z), z g^{\prime}(z), z^{2} g^{\prime \prime}(z) ; z\right) \in \Omega .
$$

The proof is completed if it can be shown that the admissibility condition for $\phi \in \Phi_{2}[\Omega, q, \mu]$ is equivalent to the admissibility condition for $\psi$ given in Definition 1.1. Note that

$$
\frac{t}{s}+1=\frac{(p+1)^{2} w-2 \mu p(p+1) v+\mu p^{2} u}{(p+1) v-p u},
$$

and hence $\psi \in \Psi_{\mu}[\Omega, q]$. By Lemma 1.3,

$$
g(z) \prec q(z) \text { or }\left[\frac{I_{p}^{\alpha} f(z)}{z^{p-1}}\right]^{\mu} \prec q(z) .
$$


If $\Omega \neq \mathbb{C}$ is a simply connected domain, then $\Omega=h(\mathbb{U})$, for some conformal mapping $h$ of $\mathbb{U}$ onto $\Omega$. In this case the class $\Phi_{2}[h(\mathbb{U}), q, \mu]$ is written as $\Phi_{2}[h, q, \mu]$. In the particular case $q(z)=M z, M>0$, the class of admissible functions $\Phi_{2}[\Omega, q, \mu]$, denoted by $\Phi_{2}[\Omega, M, \mu]$.

Proceeding similarly as in the previous section, the following result is an immediate consequence of Theorem 2.14.

Theorem 2.15. Let $\phi \in \Phi_{2}[h, q, \mu]$. If $f \in \mathcal{A}(p)$ satisfies

$$
\begin{gathered}
\phi\left(\left[\frac{I_{p}^{\alpha} f(z)}{z^{p-1}}\right]^{\mu},\left[\frac{I_{p}^{\alpha} f(z)}{z^{p-1}}\right]^{\mu} \frac{I_{p}^{\alpha-1} f(z)}{I_{p}^{\alpha} f(z)},\left[\frac{I_{p}^{\alpha} f(z)}{z^{p-1}}\right]^{\mu} \frac{I_{p}^{\alpha-2} f(z)}{I_{p}^{\alpha} f(z)}\right. \\
\left.+(\mu-1)\left[\frac{I_{p}^{\alpha} f(z)}{z^{p-1}}\right]^{\mu}\left[\frac{I_{p}^{\alpha-1} f(z)}{I_{p}^{\alpha} f(z)}\right]^{2} ; z\right) \prec h(z),
\end{gathered}
$$

then

$$
\left[\frac{I_{p}^{\alpha} f(z)}{z^{p-1}}\right]^{\mu} \prec q(z)
$$

Definition 2.16. Let $\Omega$ be a set in $\mathbb{C}$ and $M>0$. The class of admissible functions $\Phi_{2}[\Omega, M, \mu]$ consists of those functions $\phi: \mathbb{C}^{3} \times \mathbb{U} \rightarrow \mathbb{C}$ such that

$$
\phi\left(M e^{i \theta}, \frac{k+\mu p}{\mu(p+1)} M e^{i \theta}, \frac{L+\left[(1+2 \mu p) k+\mu^{2} p^{2}\right] M e^{i \theta}}{\mu(p+1)^{2}} ; z\right) \notin \Omega
$$

whenever $z \in \mathbb{U}, \theta \in \mathbb{R}, \Re\left(L e^{-i \theta}\right) \geq(k-1) k M$ for all real $\theta, p \in \mathbb{N}$ and $k \geq \mu$.

Corollary 2.17. Let $\phi \in \Phi_{2}[\Omega, M, \mu]$. If $f \in \mathcal{A}(p)$ satisfies

$$
\begin{gathered}
\phi\left(\left[\frac{I_{p}^{\alpha} f(z)}{z^{p-1}}\right]^{\mu},\left[\frac{I_{p}^{\alpha} f(z)}{z^{p-1}}\right]^{\mu} \frac{I_{p}^{\alpha-1} f(z)}{I_{p}^{\alpha} f(z)},\left[\frac{I_{p}^{\alpha} f(z)}{z^{p-1}}\right]^{\mu} \frac{I_{p}^{\alpha-2} f(z)}{I_{p}^{\alpha} f(z)}\right. \\
\left.+(\mu-1)\left[\frac{I_{p}^{\alpha} f(z)}{z^{p-1}}\right]^{\mu}\left[\frac{I_{p}^{\alpha-1} f(z)}{I_{p}^{\alpha} f(z)}\right]^{2} ; z\right) \in \Omega,
\end{gathered}
$$

then

$$
\left|\frac{I_{p}^{\alpha} f(z)}{z^{p-1}}\right|^{\mu}<M
$$

In the special case $\Omega=\{\omega:|\omega|<M\}$, the class $\Phi_{2}[\Omega, M, \mu]$ is simply denoted by $\Phi_{2}[M, \mu]$. 
Corollary 2.18. Let $\phi \in \Phi_{2}[M, \mu]$. If $f \in \mathcal{A}(p)$ satisfies

$$
\begin{gathered}
\mid \phi\left(\left[\frac{I_{p}^{\alpha} f(z)}{z^{p-1}}\right]^{\mu},\left[\frac{I_{p}^{\alpha} f(z)}{z^{p-1}}\right]^{\mu} \frac{I_{p}^{\alpha-1} f(z)}{I_{p}^{\alpha} f(z)},\left[\frac{I_{p}^{\alpha} f(z)}{z^{p-1}}\right]^{\mu} \frac{I_{p}^{\alpha-2} f(z)}{I_{p}^{\alpha} f(z)}\right. \\
\left.+(\mu-1)\left[\frac{I_{p}^{\alpha} f(z)}{z^{p-1}}\right]^{\mu}\left[\frac{I_{p}^{\alpha-1} f(z)}{I_{p}^{\alpha} f(z)}\right]^{2} ; z\right) \mid<M,
\end{gathered}
$$

then

$$
\left|\frac{I_{p}^{\alpha} f(z)}{z^{p-1}}\right|^{\mu}<M
$$

Corollary 2.19. If $k \geq \mu$ and $f \in \mathcal{A}(p)$ satisfies

$$
\left|\left\lceil\frac{I_{p}^{\alpha} f(z)}{z^{p-1}}\right]^{\mu} \frac{I_{p}^{\alpha-1} f(z)}{I_{p}^{\alpha} f(z)}\right|<M \quad(\alpha>1),
$$

then

$$
\left|\frac{I_{p}^{\alpha} f(z)}{z^{p-1}}\right|^{\mu}<M \quad(z \in \mathbb{U}) .
$$

Proof. This follows from Corollary 2.18 by taking

$$
\phi(u, v, w ; z)=v=\frac{k+\mu p}{\mu(p+1)} M e^{i \theta} .
$$

3. Superordination and sandwich results involving $I_{p}^{\alpha}$. The dual problem of differential subordination, that is, differential superordination of the integral operator $I_{p}^{\alpha}$ is investigated in this section. For this purpose the class of admissible functions is given in the following definition.

Definition 3.1. Let $\Omega$ be a set in $\mathbb{C}$ and $q \in \mathcal{H}[0, \mu p]$ with $z q^{\prime}(z) \neq 0$. The class of admissible functions $\Phi_{1}^{\prime}[\Omega, q, \mu]$ consists of those functions $\phi$ : $\mathbb{C}^{3} \times \overline{\mathbb{U}} \rightarrow \mathbb{C}$ that satisfy the admissibility condition:

$$
\phi(u, v, w ; \zeta) \in \Omega
$$

whenever

$$
\begin{gathered}
u=q(z), v=\frac{z q^{\prime}(z)+m \mu q(z)}{m \mu(p+1)}, \\
\Re\left\{\frac{(p+1)^{2} w-2 \mu(p+1) v+\mu u}{(p+1) v-u}\right\} \geq \frac{1}{m} \Re\left\{1+\frac{\zeta q^{\prime \prime}(\zeta)}{q^{\prime}(\zeta)}\right\},
\end{gathered}
$$

where $z \in \mathbb{U}, \zeta \in \partial \mathbb{U}$ and $m \geq \mu p$. 
Theorem 3.2. Let $\phi \in \Phi_{1}^{\prime}[\Omega, q, \mu]$. If $f \in \mathcal{A}(p),\left[I_{p}^{\alpha} f(z)\right]^{\mu} \in \mathcal{F}_{0}$ and

$$
\begin{aligned}
& \phi\left(\left[I_{p}^{\alpha} f(z)\right]^{\mu},\left[I_{p}^{\alpha} f(z)\right]^{\mu-1} I_{p}^{\alpha-1} f(z),\left[I_{p}^{\alpha} f(z)\right]^{\mu-1} I_{p}^{\alpha-2} f(z)\right. \\
& \left.\quad+(\mu-1)\left[I_{p}^{\alpha} f(z)\right]^{\mu-2}\left[I_{p}^{\alpha-1} f(z)\right]^{2} ; z\right)
\end{aligned}
$$

is univalent in $\mathbb{U}$, then

$$
\begin{aligned}
\Omega \subset\{ & \phi\left(\left[I_{p}^{\alpha} f(z)\right]^{\mu},\left[I_{p}^{\alpha} f(z)\right]^{\mu-1} I_{p}^{\alpha-1} f(z),\left[I_{p}^{\alpha} f(z)\right]^{\mu-1} I_{p}^{\alpha-2} f(z)\right. \\
& \left.\left.+(\mu-1)\left[I_{p}^{\alpha} f(z)\right]^{\mu-2}\left[I_{p}^{\alpha-1} f(z)\right]^{2} ; z\right): z \in \mathbb{U}\right\}
\end{aligned}
$$

implies

$$
q(z) \prec\left[I_{p}^{\alpha} f(z)\right]^{\mu} .
$$

Proof. From (2.7) and (3.1), we have

$$
\Omega \subset\left\{\psi\left(g(z), z g^{\prime}(z), z^{2} g^{\prime \prime}(z) ; z\right): z \in \mathbb{U}\right\} .
$$

From (2.5), we see that the admissibility condition for $\phi \in \Phi_{1}^{\prime}[\Omega, q, \mu]$ is equivalent to the admissibility condition for $\psi$ as given in Definition 1.2. Hence $\psi \in \Psi_{\mu p}^{\prime}[\Omega, q]$, and by Lemma 1.4,

$$
q(z) \prec g(z) \quad \text { or } \quad q(z) \prec\left[I_{p}^{\alpha} f(z)\right]^{\mu} .
$$

If $\Omega \neq \mathbb{C}$ is a simply connected domain, then $\Omega=h(\mathbb{U})$ for some conformal mapping $h$ of $\mathbb{U}$ onto $\Omega$. In this case the class $\Phi_{1}^{\prime}[h(\mathbb{U}), q, \mu]$ is written as $\Phi_{1}^{\prime}[h, q, \mu]$.

Proceeding similarly as in the previous section, the following result is an immediate consequence of Theorem 3.2.

Theorem 3.3. Let $h$ be analytic on $\mathbb{U}$ and $\phi \in \Phi_{1}^{\prime}[h, q, \mu]$. If $f \in \mathcal{A}(p)$, $\left[I_{p}^{\alpha} f(z)\right]^{\mu} \in \mathcal{F}_{0}$ and

$$
\begin{aligned}
& \phi\left(\left[I_{p}^{\alpha} f(z)\right]^{\mu},\left[I_{p}^{\alpha} f(z)\right]^{\mu-1} I_{p}^{\alpha-1} f(z),\left[I_{p}^{\alpha} f(z)\right]^{\mu-1} I_{p}^{\alpha-2} f(z)\right. \\
& \left.\quad+(\mu-1)\left[I_{p}^{\alpha} f(z)\right]^{\mu-2}\left[I_{p}^{\alpha-1} f(z)\right]^{2} ; z\right)
\end{aligned}
$$

is univalent in $\mathbb{U}$, then

$$
\begin{aligned}
h(z) \prec \phi & \left(\left[I_{p}^{\alpha} f(z)\right]^{\mu},\left[I_{p}^{\alpha} f(z)\right]^{\mu-1} I_{p}^{\alpha-1} f(z),\left[I_{p}^{\alpha} f(z)\right]^{\mu-1} I_{p}^{\alpha-2} f(z)\right. \\
& \left.+(\mu-1)\left[I_{p}^{\alpha} f(z)\right]^{\mu-2}\left[I_{p}^{\alpha-1} f(z)\right]^{2} ; z\right)
\end{aligned}
$$

implies

$$
q(z) \prec\left[I_{p}^{\alpha} f(z)\right]^{\mu}
$$


Theorems 3.2 and 3.3 can only be used to obtain subordinants of differential superordination of the form (3.1) or (3.2). The following theorem proves the existence of the best subordinant of (3.2) for certain $\phi$.

Theorem 3.4. Let $h$ be analytic in $\mathbb{U}$ and $\phi: \mathbb{C}^{3} \times \overline{\mathbb{U}} \rightarrow \mathbb{C}$. Suppose that the differential equation

$$
\phi\left(q(z), z q^{\prime}(z), z^{2} q^{\prime \prime}(z) ; z\right)=h(z)
$$

has a solution $q \in \mathcal{F}_{0}$. If $\phi \in \Phi_{1}^{\prime}[h, q, \mu], f \in \mathcal{A}(p),\left[I_{p}^{\alpha} f(z)\right]^{\mu} \in \mathcal{F}_{0}$ and

$$
\begin{aligned}
\phi\left(\left[I_{p}^{\alpha} f(z)\right]^{\mu},\left[I_{p}^{\alpha} f(z)\right]^{\mu-1} I_{p}^{\alpha-1} f(z),\left[I_{p}^{\alpha} f(z)\right]^{\mu-1} I_{p}^{\alpha-2} f(z)\right. \\
\left.+(\mu-1)\left[I_{p}^{\alpha} f(z)\right]^{\mu-2}\left[I_{p}^{\alpha-1} f(z)\right]^{2} ; z\right)
\end{aligned}
$$

is univalent in $\mathbb{U}$, then

$$
\begin{aligned}
h(z) \prec \phi & \left(\left[I_{p}^{\alpha} f(z)\right]^{\mu},\left[I_{p}^{\alpha} f(z)\right]^{\mu-1} I_{p}^{\alpha-1} f(z),\left[I_{p}^{\alpha} f(z)\right]^{\mu-1} I_{p}^{\alpha-2} f(z)\right. \\
& \left.+(\mu-1)\left[I_{p}^{\alpha} f(z)\right]^{\mu-2}\left[I_{p}^{\alpha-1} f(z)\right]^{2} ; z\right)
\end{aligned}
$$

implies

$$
q(z) \prec\left[I_{p}^{\alpha} f(z)\right]^{\mu} .
$$

and $q$ is the best subordinant.

Proof. The proof is similar to the proof of Theorem 2.6 and is therefore omitted.

Combining Theorems 2.3 and 3.3, we obtain the following sandwich-type theorem.

Corollary 3.5. Let $h_{1}$ and $q_{1}$ be analytic functions in $\mathbb{U}, h_{2}$ be univalent function in $\mathbb{U}, q_{2} \in \mathcal{F}_{0}$ with $q_{1}(0)=q_{2}(0)=0$ and $\phi \in \Phi_{1}\left[h_{2}, q_{2}, \mu\right] \cap$ $\Phi_{1}^{\prime}\left[h_{1}, q_{1}, \mu\right]$. If $f \in \mathcal{A}(p),\left[I_{p}^{\alpha} f(z)\right]^{\mu} \in \mathcal{H}[0, \mu p] \cap \mathcal{F}_{0}$ and

$$
\begin{aligned}
& \phi\left(\left[I_{p}^{\alpha} f(z)\right]^{\mu},\left[I_{p}^{\alpha} f(z)\right]^{\mu-1} I_{p}^{\alpha-1} f(z),\left[I_{p}^{\alpha} f(z)\right]^{\mu-1} I_{p}^{\alpha-2} f(z)\right. \\
& \left.\quad+(\mu-1)\left[I_{p}^{\alpha} f(z)\right]^{\mu-2}\left[I_{p}^{\alpha-1} f(z)\right]^{2} ; z\right)
\end{aligned}
$$

is univalent in $\mathbb{U}$, then

$$
\begin{aligned}
h_{1}(z) \prec \phi & \left(\left[I_{p}^{\alpha} f(z)\right]^{\mu},\left[I_{p}^{\alpha} f(z)\right]^{\mu-1} I_{p}^{\alpha-1} f(z),\left[I_{p}^{\alpha} f(z)\right]^{\mu-1} I_{p}^{\alpha-2} f(z)\right. \\
& \left.+(\mu-1)\left[I_{p}^{\alpha} f(z)\right]^{\mu-2}\left[I_{p}^{\alpha-1} f(z)\right]^{2} ; z\right) \prec h_{2}(z),
\end{aligned}
$$

implies

$$
q_{1}(z) \prec\left[I_{p}^{\alpha} f(z)\right]^{\mu} \prec q_{2}(z)
$$


Definition 3.6. Let $\Omega$ be a set in $\mathbb{C}$ and $q \in \mathcal{H}[0, \mu]$ with $z q^{\prime}(z) \neq 0$. The class of admissible functions $\Phi_{2}^{\prime}[\Omega, q, \mu]$ consists of those functions $\phi$ : $\mathbb{C}^{3} \times \overline{\mathbb{U}} \rightarrow \mathbb{C}$ that satisfy the admissibility condition:

$$
\phi(u, v, w ; \zeta) \in \Omega
$$

whenever

$$
\begin{gathered}
u=q(z), v=\frac{z q^{\prime}(z)+m \mu p q(z)}{m \mu(p+1)}, \\
\Re\left\{\frac{(p+1)^{2} w-2 \mu p(p+1) v+\mu p^{2} u}{(p+1) v-p u}\right\} \geq \frac{1}{m} \Re\left\{1+\frac{\zeta q^{\prime \prime}(\zeta)}{q^{\prime}(\zeta)}\right\},
\end{gathered}
$$

where $z \in \mathbb{U}, \zeta \in \partial \mathbb{U}$ and $m \geq \mu$.

Now we will give the dual result of Theorem 2.14 for differential superordination.

Theorem 3.7. Let $\phi \in \Phi_{2}^{\prime}[\Omega, q, \mu]$. If $f \in \mathcal{A}(p),\left[\frac{I_{p}^{\alpha} f(z)}{z^{p-1}}\right]^{\mu} \in \mathcal{F}_{0}$ and

$$
\begin{aligned}
& \phi\left(\left[\frac{I_{p}^{\alpha} f(z)}{z^{p-1}}\right]^{\mu},\left[\frac{I_{p}^{\alpha} f(z)}{z^{p-1}}\right]^{\mu} \frac{I_{p}^{\alpha-1} f(z)}{I_{p}^{\alpha} f(z)},\left[\frac{I_{p}^{\alpha} f(z)}{z^{p-1}}\right]^{\mu} \frac{I_{p}^{\alpha-2} f(z)}{I_{p}^{\alpha} f(z)}\right. \\
& \left.+(\mu-1)\left[\frac{I_{p}^{\alpha} f(z)}{z^{p-1}}\right]^{\mu}\left[\frac{I_{p}^{\alpha-1} f(z)}{I_{p}^{\alpha} f(z)}\right]^{2} ; z\right),
\end{aligned}
$$

is univalent in $\mathbb{U}$, then

$$
\begin{aligned}
\Omega \subset\{\phi & \left(\left[\frac{I_{p}^{\alpha} f(z)}{z^{p-1}}\right]^{\mu},\left[\frac{I_{p}^{\alpha} f(z)}{z^{p-1}}\right]^{\mu} \frac{I_{p}^{\alpha-1} f(z)}{I_{p}^{\alpha} f(z)},\left[\frac{I_{p}^{\alpha} f(z)}{z^{p-1}}\right]^{\mu} \frac{I_{p}^{\alpha-2} f(z)}{I_{p}^{\alpha} f(z)}\right. \\
& \left.\left.+(\mu-1)\left[\frac{I_{p}^{\alpha} f(z)}{z^{p-1}}\right]^{\mu}\left[\frac{I_{p}^{\alpha-1} f(z)}{I_{p}^{\alpha} f(z)}\right]^{2} ; z\right): z \in \mathbb{U}\right\},
\end{aligned}
$$

implies

$$
q(z) \prec\left[\frac{I_{p}^{\alpha} f(z)}{z^{p-1}}\right]^{\mu}
$$

Proof. From (2.17) and (3.4), we have

$$
\Omega \subset\left\{\psi\left(g(z), z g^{\prime}(z), z^{2} g^{\prime \prime}(z) ; z\right): z \in \mathbb{U}\right\} .
$$

From (2.15), we see that the admissibility condition for $\phi \in \Phi_{2}^{\prime}[\Omega, q, \mu]$ is equivalent to the admissibility condition for $\psi$ as given in Definition 1.2. Hence $\psi \in \Psi^{\prime}[\Omega, q]$, and by Lemma 1.4 ,

$$
q(z) \prec g(z) \text { or } q(z) \prec\left[\frac{I_{p}^{\alpha} f(z)}{z^{p-1}}\right]^{\mu} .
$$


If $\Omega \neq \mathbb{C}$ is a simply connected domain and $\Omega=h(\mathbb{U})$ for some conformal mapping $h$ of $\mathbb{U}$ onto $\Omega$, then the class $\Phi_{2}^{\prime}[h(\mathbb{U}), q, \mu]$ is written as $\Phi_{2}^{\prime}[h, q, \mu]$.

Proceeding similarly as in the previous section, the following result is an immediate consequence of Theorem 3.7.

Theorem 3.8. Let $q \in \mathcal{H}[0, \mu], h$ is analytic on $\mathbb{U}$ and $\phi \in \Phi_{2}^{\prime}[h, q, \mu]$. If $f \in \mathcal{A}(p),\left[\frac{I_{p}^{\alpha} f(z)}{z^{p-1}}\right]^{\mu} \in \mathcal{F}_{0}$ and

$$
\begin{aligned}
& \phi\left(\left[\frac{I_{p}^{\alpha} f(z)}{z^{p-1}}\right]^{\mu},\left[\frac{I_{p}^{\alpha} f(z)}{z^{p-1}}\right]^{\mu} \frac{I_{p}^{\alpha-1} f(z)}{I_{p}^{\alpha} f(z)},\left[\frac{I_{p}^{\alpha} f(z)}{z^{p-1}}\right]^{\mu} \frac{I_{p}^{\alpha-2} f(z)}{I_{p}^{\alpha} f(z)}\right. \\
& \left.+(\mu-1)\left[\frac{I_{p}^{\alpha} f(z)}{z^{p-1}}\right]^{\mu}\left[\frac{I_{p}^{\alpha-1} f(z)}{I_{p}^{\alpha} f(z)}\right]^{2} ; z\right),
\end{aligned}
$$

is univalent in $\mathbb{U}$, then

$$
\begin{aligned}
h(z) \prec \phi & \left(\left[\frac{I_{p}^{\alpha} f(z)}{z^{p-1}}\right]^{\mu},\left[\frac{I_{p}^{\alpha} f(z)}{z^{p-1}}\right]^{\mu} \frac{I_{p}^{\alpha-1} f(z)}{I_{p}^{\alpha} f(z)},\left[\frac{I_{p}^{\alpha} f(z)}{z^{p-1}}\right]^{\mu} \frac{I_{p}^{\alpha-2} f(z)}{I_{p}^{\alpha} f(z)}\right. \\
& \left.+(\mu-1)\left[\frac{I_{p}^{\alpha} f(z)}{z^{p-1}}\right]^{\mu}\left[\frac{I_{p}^{\alpha-1} f(z)}{I_{p}^{\alpha} f(z)}\right]^{2} ; z\right),
\end{aligned}
$$

implies

$$
q(z) \prec\left[\frac{I_{p}^{\alpha} f(z)}{z^{p-1}}\right]^{\mu} .
$$

Combining Theorems 2.15 and 3.8, we obtain the following sandwich-type theorem.

Corollary 3.9. Let $h_{1}$ and $q_{1}$ be analytic functions in $\mathbb{U}, h_{2}$ be univalent function in $\mathbb{U}, q_{2} \in \mathcal{F}_{0}$ with $q_{1}(0)=q_{2}(0)=0$ and $\phi \in \Phi_{2}\left[h_{2}, q_{2}, \mu\right] \cap$ $\Phi_{2}^{\prime}\left[h_{1}, q_{1}, \mu\right]$. If $f \in \mathcal{A}(p),\left[\frac{I_{p}^{\alpha} f(z)}{z^{p-1}}\right]^{\mu} \in \mathcal{H}[0, \mu] \cap \mathcal{F}_{0}$ and

$$
\begin{gathered}
\phi\left(\left[\frac{I_{p}^{\alpha} f(z)}{z^{p-1}}\right]^{\mu},\left[\frac{I_{p}^{\alpha} f(z)}{z^{p-1}}\right]^{\mu} \frac{I_{p}^{\alpha-1} f(z)}{I_{p}^{\alpha} f(z)},\left[\frac{I_{p}^{\alpha} f(z)}{z^{p-1}}\right]^{\mu} \frac{I_{p}^{\alpha-2} f(z)}{I_{p}^{\alpha} f(z)}\right. \\
\left.+(\mu-1)\left[\frac{I_{p}^{\alpha} f(z)}{z^{p-1}}\right]^{\mu}\left[\frac{I_{p}^{\alpha-1} f(z)}{I_{p}^{\alpha} f(z)}\right]^{2} ; z\right)
\end{gathered}
$$

is univalent in $\mathbb{U}$, then 


$$
\begin{aligned}
h_{1}(z) \prec \phi & \left(\left[\frac{I_{p}^{\alpha} f(z)}{z^{p-1}}\right]^{\mu},\left[\frac{I_{p}^{\alpha} f(z)}{z^{p-1}}\right]^{\mu} \frac{I_{p}^{\alpha-1} f(z)}{I_{p}^{\alpha} f(z)},\left[\frac{I_{p}^{\alpha} f(z)}{z^{p-1}}\right]^{\mu} \frac{I_{p}^{\alpha-2} f(z)}{I_{p}^{\alpha} f(z)}\right. \\
& \left.+(\mu-1)\left[\frac{I_{p}^{\alpha} f(z)}{z^{p-1}}\right]^{\mu}\left[\frac{I_{p}^{\alpha-1} f(z)}{I_{p}^{\alpha} f(z)}\right]^{2} ; z\right) \prec h_{2}(z)
\end{aligned}
$$

implies

$$
q_{1}(z) \prec\left[\frac{I_{p}^{\alpha} f(z)}{z^{p-1}}\right]^{\mu} \prec q_{2}(z) .
$$

Remark 3.10. Putting $\mu=1$ in our results, we obtain the results of Aouf and Seoudy [6, Theorems 1 and 2, Corollary 1, Theorems 3 and 4, Corollaries 2, 3, and 4, Theorems 5 and 6, Corollaries 5, 6, and 7, Theorems 9, 10, and 11, Corollary 10, Theorem 12 and 13 and Corollary 11, respectively].

\section{REFERENCES}

[1] Aghalary, R., Ali, R. M., Joshi, S. B., Ravichandran, V., Inequalities for analytic functions defined by certain linear operator, Internat. J. Math. Sci. 4 (2) (2005), $267-274$.

[2] Ali, R. M., Ravichandran, V., Seenivasagan, N., Differential subordination and superodination of analytic functions defined by the multiplier transformation, Math. Inequal. Appl. 12 (1) (2009), 123-139.

[3] Aouf, M. K., Inequalities involving certain integral operator, J. Math. Inequal. 2 (2) (2008), 537-547.

[4] Aouf, M. K., Hossen, H. M., Lashin, A. Y., An application of certain integral operators, J. Math. Anal. Appl. 248 (2) (2000), 475-481.

[5] Aouf, M. K., Seoudy, T. M., Differential subordination and superordination of analytic functions defined by an integral operator, European J. Pure Appl. Math. 3 (1) (2010), 26-44.

[6] Aouf, M. K., Seoudy, T. M., Differential subordination and superordination of analytic functions defined by certain integral operator, Acta Univ. Apulensis 24 (2010), 211-229.

[7] Bulboacă, T., Differential Subordinations and Superordinations. Recent Results, House of Scientific Book Publ., Cluj-Napoca, 2005.

[8] Kim, Y. C., Srivastava, H. M., Inequalities involving certain families of integral and convolution operators, Math. Inequal. Appl. 7 (2) (2004), 227-234.

[9] Jung, T. B., Kim, Y. C., Srivastava, H. M., The Hardy space of analytic functions associated with certain one-parameter families of integral operators, J. Math. Anal. Appl. 176 (1993), 138-147.

[10] Miller, S. S., Mocanu, P. T., Differential Subordinations: Theory and Applications, Marcel Dekker, New York-Basel, 2000.

[11] Miller, S. S., Mocanu, P. T., Subordinants of differential superordinations, Complex Var. Theory Appl. 48 (10) (2003), 815-826.

[12] Shams, S., Kulkarni, S. R., Jahangir, Jay M., Subordination properties for p-valent functions defined by integral operators, Internat. J. Math. Math. Sci. Vol. 2006, Article ID $94572,1-3$. 
T. M. Seoudy

Department of Mathematics

Faculty of Science

Fayoum University

Fayoum 63514

Egypt

Jamoum University College

Umm Al-Qura University

Makkah

Saudi Arabia

e-mail: tms00@fayoum.edu.eg

Received October 14, 2018
M. K. Aouf

Department of Mathematics

Faculty of Science

Mansoura University

Mansoura 35516

Egypt

e-mail: mkaouf 127@yahoo.com 